\title{
Geometric evolutions driven by threshold dynamics
}

\author{
Minsu SONG ${ }^{\dagger}$ \\ Department of Mathematics, University of Illinois at Urbana-Champaign, USA \\ Current address: Department of Mathematics, Pohang University of Science and Technology, \\ Pohang 790-784, Kyungpook, Korea
}

[Received 22 October 2004 and in revised form 2 May 2005]

\begin{abstract}
We study threshold dynamics on $\mathbb{R}^{n}$ which satisfies monotonicity, translation invariance and finite propagation speed. We develop general schemes for the convergence of threshold dynamics to geometric evolutions governed by a velocity function depending on the normal direction alone.
\end{abstract}

2000 Mathematics Subject Classification: Primary 35A05; Secondary 35A30, 35D05.

Keywords: Geometric evolution; threshold dynamics; viscosity solution.

\section{Introduction}

Consider the evolution of interfaces (hypersurfaces) in $\mathbb{R}^{n}$ which propagate in the normal direction with the velocity

$$
V=v(\mathbf{n}, D \mathbf{n}),
$$

where $\mathbf{n}$ and $D \mathbf{n}$ are the unit normal vector to the surface and its gradient respectively. The study of the evolving interfaces is very interesting since (1.1) arises in many areas, such as phase transitions, image processing, pattern formation, turbulent flame propagation, etc. Typical examples are anisotropic motions with the normal velocity

$$
V=-\operatorname{tr}[S(\mathbf{n}) D \mathbf{n}]+v(\mathbf{n})
$$

where $S(\cdot)$ is a continuous function taking values in the space $\mathcal{S}^{n}$ of $n \times n$ symmetric matrices and $v$ is real-valued. Special cases of $(1.2)$ are motion by curvature, corresponding to $S(\mathbf{n}) \equiv I$ and $v(\mathbf{n}) \equiv 0$, and curvature independent motions, corresponding to $S(\mathbf{n}) \equiv 0$. Solutions of these geometric evolution problems typically develop singularities, regardless of the smoothness of the initial data (see [12], [14]).

To overcome such difficulties, the level set approach was introduced by Chen, Giga, and Goto [8] and independently by Evans and Spruck [10]. In [10] motion by mean curvature is dealt with while in [8] more general geometric motions are discussed. Their ideas are based on considering interfaces as level sets of the solution of a degenerate parabolic partial differential equation.

One approach to (1.1) is that some classes of dynamics which model the microscopic behavior of physical phenomena (for example, threshold dynamics, cellular automata) provide approximate schemes for (1.1). Among others, Bence, Merriman, and Osher [6] first proposed a simple approximation scheme (BMO) for motion by mean curvature. Later, Evans [9] and Barles and Georgelin [2] gave the first analysis of the BMO algorithm. More recently, Ishii, Pires,

†E-mail: msong@postech.ac.kr 
and Souganidis [13] further extended the BMO scheme to anisotropic motions with the normal velocity (1.2). These schemes are generalizations and extensions of the threshold dynamics models introduced by Gravner and Griffeath [11] to study cellular automaton modeling of excitable media.

More precisely, Ishii, Pires, and Souganidis [13] considered the following: Fix a threshold number $\theta \in(0,1)$ and choose a measurable real-valued function $f$ on $\mathbb{R}^{n}$ such that

$$
\int_{\mathbb{R}^{n}} f(x) \mathrm{d} x=1 \text { and } f \geqslant 0 \quad \text { on } \mathbb{R}^{n} .
$$

For $h>0, \alpha \in \mathbb{R}$, and a subset $G$ of $\mathbb{R}^{n}$, define

$$
F_{h}(G)=\left\{x \in \mathbb{R}^{n}: \int_{\mathbb{R}^{n}} f(y) \chi_{G}\left(x-h^{\alpha} y\right) \mathrm{d} y \geqslant \theta\right\},
$$

where $\chi_{G}$ is the characteristic function of the set $G$. By choosing the appropriate function $f$ and the values $\alpha$ and $\theta$, they showed that $F_{h}^{k}(G)$, the $k$-th iterate of $F_{h}(G)$, converges, as $k h \rightarrow t$, to $\Omega(t)$ in a suitable sense, where $\Omega(t)$ with $\Omega(0)=G$ is an evolution with normal velocity of the type 1.2. In particular, if $\alpha=\theta=1 / 2$ and $f$ is a Gaussian, then $F_{h}^{k}(G)$ approximates motion by mean curvature

$$
V=-\operatorname{tr}(D \mathbf{n}) \text {. }
$$

This is the scheme first proposed by Bence, Merriman, and Osher [6] and analyzed by Evans [9] and Barles and Georgelin [2].

\subsection{The main results}

In this study, we consider a class of threshold dynamics with the following properties: For $h>0$, suppose that $F_{h}$ is a mapping from the set of open subsets in $\mathbb{R}^{n}$ to itself such that

(F1) Monotonicity: If $G \subset H \subset \mathbb{R}^{n}$, then $F_{h} G \subset F_{h} H$;

(F2) Translation invariance: $F_{h}\left(\tau_{y} G\right)=\tau_{y}\left(F_{h} G\right)$ for all $y \in \mathbb{R}^{n}$, where $\tau_{y} G=\{y+x$ : $x \in G\}, G \subset \mathbb{R}^{n}$;

(F3) Finite propagation speed: There exists $c>0$ such that if $G \cap B_{2 c h}(x)=H \cap B_{2 c h}(x)$, then

$$
F_{h} G \cap B_{c h}(x)=F_{h} H \cap B_{c h}(x),
$$

where $B_{r}(x)=\left\{y \in \mathbb{R}^{n}:|y-x|<r\right\}$.

Notice that if we choose $\alpha=1, \theta \in(0,1)$, and $f$ a measurable real-valued function on $\mathbb{R}^{n}$ with a compact support, then $F_{h}$ in 1.3 satisfies (F1)-(F3).

One way to generate a limiting process from a family $\left\{F_{h}\right\}$ as above is to define, for $t>0$ and an open subset $G$ in $\mathbb{R}^{n}$,

and

$$
\underline{\mathcal{A}}(t) G=\left(\bigcup_{G^{\prime} \subset \subset G} \liminf _{h \rightarrow 0} F_{h}^{\lfloor t / h\rfloor} G^{\prime}\right)^{o}
$$

$$
\overline{\mathcal{A}}(t) G=\left(\bigcap_{G^{\prime} \supset \supset G} \limsup _{h \rightarrow 0} F_{h}^{\lfloor t / h\rfloor} G^{\prime}\right)^{o},
$$

where $A^{o}$ denotes the interior of the set $A$ and $\lfloor s\rfloor$ is the greatest integer which does not exceed $s$. In view of (F1)-(F3), one can naturally consider the properties for the evolutions $\underline{\mathcal{A}}(t), \overline{\mathcal{A}}(t)$ : Suppose that for every $t>0, \mathcal{A}(t)$ is a mapping from the set of all open subsets of $\mathbb{R}^{n}$ to itself such that 
(A1) Semigroup: $\mathcal{A}(t+s)=\mathcal{A}(t) \mathcal{A}(s)$ for all $s, t \geqslant 0$;

(A2) Translation invariance: $\mathcal{A}(t) \tau_{y}=\tau_{y} \mathcal{A}(t)$ for all $t \geqslant 0$ and $y \in \mathbb{R}^{n}$;

(A3) Monotonicity: If $G \subset H$, then $\mathcal{A}(t) G \subset \mathcal{A}(t) H$;

(A4) Finite propagation speed: $\mathcal{A}(t) \emptyset=\emptyset, \mathcal{A}(t) \mathbb{R}^{n}=\mathbb{R}^{n}$ for all $t>0$. In addition, there exists $c>0$ such that if $G \cap B_{c t}(x)=H \cap B_{c t}(x)$, then

$$
(\mathcal{A}(s) G) \cap B_{c(t-s)}(x)=(\mathcal{A}(s) H) \cap B_{c(t-s)}(x), \quad \forall s \leqslant t .
$$

Then we have the following:

TheOREm 1.1 Assume $\mathcal{A}_{1}(t), \mathcal{A}_{2}(t)$ satisfy (A2)-(A4) and, for all $t, s>0$,

$$
\mathcal{A}_{1}(t) \mathcal{A}_{1}(s) \subset \mathcal{A}_{1}(t+s), \quad \mathcal{A}_{2}(t) \mathcal{A}_{2}(s) \supset \mathcal{A}_{2}(t+s) .
$$

Then there exist continuous functions $v_{1}, v_{2}: S^{n-1} \rightarrow \mathbb{R}$ such that for every open set $G$, $\left\{\mathcal{A}_{1}(t) G\right\}_{t \geqslant 0}$ is a supersolution of $V=v_{1}(\mathbf{n})$ and $\left\{\mathcal{A}_{2}(t) G\right\}_{t} \geqslant 0$ is a subsolution of $V=v_{2}(\mathbf{n})$. In addition, if $\mathcal{A}(t)$ satisfies (A1)-(A4), then there exists a continuous function $v: S^{n-1} \rightarrow \mathbb{R}$ such that $\{\mathcal{A}(t) G\}_{t} \geqslant 0$ is a weak solution of $V=v(\mathbf{n})$.

The definitions of subsolution, supersolution, and weak solution are given in Definition 3.1

In view of nonlinear semigroup theory, Theorem 1.1 says that relaxed semigroup properties with (A2)-(A4) ensure the existence of "one-sided infinitesimal generators", which coincide if the semigroup property (A1) holds.

Regarding the properties (A1)-(A4), Barles and Souganidis [4] and Biton [7] already studied these kinds of semigroups acting on sets in a more general setting, which is motivated in part by the axiomatic approach to image processing developed by Alvarez, Guichard, Lions, and Morel in [1]. But we remark that Theorem 1.1 cannot be derived directly from the result of Barles and Souganidis since here we do not assume what they call "locality and regularity", which is more or less the existence of a velocity function $v$.

Note that because of the condition (A4), the limit motions are necessarily of order one, which essentially excludes the mean curvature motion. It turns out that they are also of local nature. This is due to the fact that the scheme is defined for any set, even unbounded. Interestingly, this can be interpreted as a negative statement: one cannot expect to approximate second order or nonlocal evolution motions by using threshold dynamics which are monotone, translation invariant, with finite propagation speed and defined for any set.

Our main result, using Theorem 1.1 , is the following:

THEOREM $1.2 \mathcal{A}(t)$ and $\overline{\mathcal{A}}(t)$ satisfy conditions (A2)-(A4). Moreover, for all $s, t \geqslant 0$, we have

$$
\underline{\mathcal{A}}(t) \underline{\mathcal{A}}(s) \subset \underline{\mathcal{A}}(t+s), \quad \overline{\mathcal{A}}(t) \overline{\mathcal{A}}(s) \supset \overline{\mathcal{A}}(t+s) .
$$

Consequently, there exist two continuous functions $\underline{v} \leqslant \bar{v}$ from $S^{n-1}$ to $\mathbb{R}$ such that for every open set $G,\{\underline{\mathcal{A}}(t) G\}_{t} \geqslant 0$ is a supersolution of $V=\underline{v}(\mathbf{n})$ and $\{\overline{\mathcal{A}}(t) G\}_{t} \geqslant 0$ is a subsolution of $V=\bar{v}(\mathbf{n})$.

To ensure $\underline{v}=\bar{v}$, we further assume

(F4) For any $s \leqslant h$ and $\mathbf{n} \in S^{n-1}$, there exists $C>0$ such that

$$
\rho\left(F_{h} H_{\mathbf{n}}, F_{s}^{\lfloor h / s\rfloor} H_{\mathbf{n}}\right) \leqslant C|h-\lfloor h / s\rfloor s|,
$$


where $\rho(A, B)$ is the distance between sets $A$ and $B$ in the Hausdorff metric, and $H_{\mathbf{n}}=\left\{x \in \mathbb{R}^{n}\right.$ : $x \cdot \mathbf{n}<0\}$. The condition (F4) is not restrictive, in fact, any family $\left\{F_{h}\right\}$ of operators as in (1.3) with $\alpha=1$ satisfies (F4).

The method used in [13], to show the convergence of threshold dynamics approximation scheme to geometric evolution, relies on a certain convergence theorem [3] for monotone approximations to viscosity solutions. Therefore the authors have to invoke the level set approach. Our result bypasses this machinery and works directly with evolving sets and their discrete approximations. This shows that if a certain threshold dynamics satisfies the conditions (F1)-(F3), then one can analyze its limiting dynamics without introducing the auxiliary level set functions and appealing to the well developed machinery of viscosity solutions, as is typically done in the level set approach. We also point out that some threshold dynamics satisfying (F1)-(F3) do not admit the convolution generated from (1.3), and thus our method covers some classes of threshold dynamics not being dealt with in $[13]$.

\subsection{Outline of the paper}

This paper is organized in the following way. In Section 2, we list some notation which will be used throughout the paper. In Section 3, we define the notion of weak solutions for geometric evolutions, and study some properties of weak solutions. Section 4 is devoted to the proof of Theorem 1.1. Finally, we consider the threshold dynamics in Section 5. We present Theorem 1.2 and discuss the existence of a limiting geometric evolution obtained by iteration of the threshold dynamics.

\section{Notation}

We introduce some notation which will be used throughout this paper. We write $\mathbb{R}^{n}$ for $n$ dimensional Euclidean space and $\mathbb{R}=\mathbb{R}^{1}$. We denote generic points in $\mathbb{R}^{n}$ by $x, y, \ldots$, where $x=\left(x_{1}, \ldots, x_{n}\right)$ and so on. We write $e_{i}=(0, \ldots, 0,1, \ldots, 0)$ for the $i$-th standard unit vector.

For $x, y \in \mathbb{R}^{n}, x \cdot y$ is the usual inner product of $x$ and $y$, and $|x|=\sqrt{x \cdot x}$. For $x \in \mathbb{R}^{n}$ and $r>0$, we set $B_{r}(x)=\left\{y \in \mathbb{R}^{n}:|y-x|<r\right\}$, an open ball in $\mathbb{R}^{n}$ with center $x$ and radius $r>0$, and $S^{n-1}=\left\{x \in \mathbb{R}^{n}:|x|=1\right\}$, the $(n-1)$-dimensional unit sphere. We denote by $H_{\mathbf{n}}=\left\{x \in \mathbb{R}^{n}: x \cdot \mathbf{n}<a\right\}$ a generic half-plane in $\mathbb{R}^{n}$ with normal $\mathbf{n} \in S^{n-1}$ for some fixed but arbitrary $a \in \mathbb{R}$.

We normally use $G, H, \ldots$ to denote open subsets of $\mathbb{R}^{n}$. For $G \subset \mathbb{R}^{n}$, we write $G^{o}, \bar{G}$ and $\partial G$ for the interior, closure, and boundary of $G$, respectively. We often write $G \subset H$ in $U$ to mean $G \cap U \subset H \cap U$.

Let $G \subset \mathbb{R}^{n} \times(0, \infty)$ be open. We say $\partial G$ is $C^{k}$ at $\left(x_{0}, t_{0}\right)$ if there exist $r>0$ and a $C^{k}$ function $g: \mathbb{R}^{n-1} \times(0, \infty) \rightarrow \mathbb{R}$ such that, upon relabeling and reorienting the coordinates if necessary, we have

$$
G \cap B_{r}\left(x_{0}, t_{0}\right)=\left\{(x, t) \in B_{r}\left(x_{0}, t_{0}\right): x_{n}>g\left(x_{1}, \ldots, x_{n-1}, t\right)\right\} .
$$

We say that $\partial G$ is $C^{k}$ if $\partial G$ is $C^{k}$ at $(x, t)$ for all $(x, t) \in \partial G$ with $t>0$. Likewise, $\partial G$ is $C^{\infty}$ if $\partial G$ is $C^{k}$ for $k=1,2, \ldots$ We say a family $\{C(t)\}_{t \geqslant 0}$ of sets is a smoothly evolving set in $\mathbb{R}^{n}$ if $\partial\left(\bigcup_{t>0} C(t) \times\{t\}\right)$ as a subset of $\mathbb{R}^{n} \times(0, \infty)$ is smooth.

Given $G \subset \mathbb{R}^{n}$ and $y \in \mathbb{R}^{n}$ we denote by $\tau_{y} G$ the translation of $G$ by $y$, that is, $\tau_{y} G=\{x+y$ : $x \in G\}$. We write $\chi_{G}$ for the characteristic function of $G$, that is, $\chi_{G}(x)=1$ if $x \in G$ and 0 otherwise. Notice that $\tau_{y} \chi_{G}=\chi_{\tau_{y} G}$ for $y \in \mathbb{R}^{n}$. The support of a function $\phi$ is denoted by $\operatorname{spt} \phi$. 
We denote by BUC $\left(\mathbb{R}^{n}\right)$ the space of bounded uniformly continuous functions on $\mathbb{R}^{n}$. For $x \in \mathbb{R}^{n}$ and $G \subset \mathbb{R}^{n}$, we write $\operatorname{dist}(x, G)$ for the distance from $x$ to $G$. We denote by $\rho(G, H)$ the distance between $G$ and $H$ in the Hausdorff metric, i.e.

$$
\rho(G, H)=\max \{\operatorname{dist}(x, H), \operatorname{dist}(y, G): x \in G, y \in H\} .
$$

Given an open subset $G \subset \mathbb{R}^{n}$, we define $d_{G} \in \mathrm{BUC}\left(\mathbb{R}^{n}\right)$ as follows:

$$
d_{G}(x)= \begin{cases}\operatorname{dist}(x, \partial G), & x \in G, \operatorname{dist}(x, \partial G) \leqslant 1, \\ -\operatorname{dist}(x, \partial G), & x \notin G, \operatorname{dist}(x, \partial G) \leqslant 1, \\ 1, & x \in G, \operatorname{dist}(x, \partial G) \geqslant 1, \\ -1, & \text { otherwise. }\end{cases}
$$

\section{Weak solutions for geometric evolution problems}

In this section, we study geometric evolutions satisfying (A1)-(A4). To study evolutions of open sets, we first define the notion of subsolutions and supersolutions for geometric evolution problems, and we find some properties of weak solutions.

We start by introducing the following definition which is motivated by the paper of Bellettini and Novaga [5].

DEFinition 3.1 A family $\{G(t)\}_{t} \geqslant 0$ of open sets is a supersolution of the geometric evolution problem $V=v(\mathbf{n})$ if, whenever $t \mapsto C(t)$ is a smoothly evolving set in $\mathbb{R}^{n}$ and

(1) $C(t) \subset G(t)$ for all $t \in\left(t_{0}-\epsilon, t_{0}\right]$ for small $\epsilon>0$,

(2)

$$
\partial C(t) \cap \partial G(t)= \begin{cases}\left\{x_{0}\right\} & \text { if } t=t_{0}, \\ \varnothing & \text { if } t \neq t_{0},\end{cases}
$$

then

$$
k_{\partial C(t)}\left(x_{0}, t_{0}\right) \geqslant v(\mathbf{n}),
$$

where $\mathbf{n}$ is normal to $\partial C\left(t_{0}\right)$ at $x_{0}$ and $k_{\partial C(t)}\left(x_{0}, t_{0}\right)$ is the normal velocity of $\partial C(t)$ at $\left(x_{0}, t_{0}\right)$. Similarly, a family $\{G(t)\}_{t \geqslant 0}$ of open sets is a subsolution of the geometric evolution problem $V=v(\mathbf{n})$ if, whenever $t \mapsto C(t)$ is a smoothly evolving set in $\mathbb{R}^{n}$ and

(1) $C(t) \supset G(t)$ for all $t \in\left[t_{0}, t_{0}+\epsilon\right)$ for small $\epsilon>0$,

(2)

$$
\partial C(t) \cap \partial G(t)= \begin{cases}\left\{x_{0}\right\} & \text { if } t=t_{0}, \\ \varnothing & \text { if } t \neq t_{0},\end{cases}
$$

then

$$
k_{\partial C(t)}\left(x_{0}, t_{0}\right) \leqslant v(\mathbf{n}),
$$

where $\mathbf{n}$ is normal to $\partial C\left(t_{0}\right)$ at $x_{0}$. Finally, a family of open sets $\{G(t)\}_{t \geqslant 0}$ is a weak solution of $V=v(\mathbf{n})$ if it is both a subsolution and a supersolution of $V=v(\mathbf{n})$.

REMARK 3.2 The condition (2) in Definition 3.1 can be replaced by

$$
x_{0} \in \partial C\left(t_{0}\right) \cap \partial G\left(t_{0}\right) .
$$



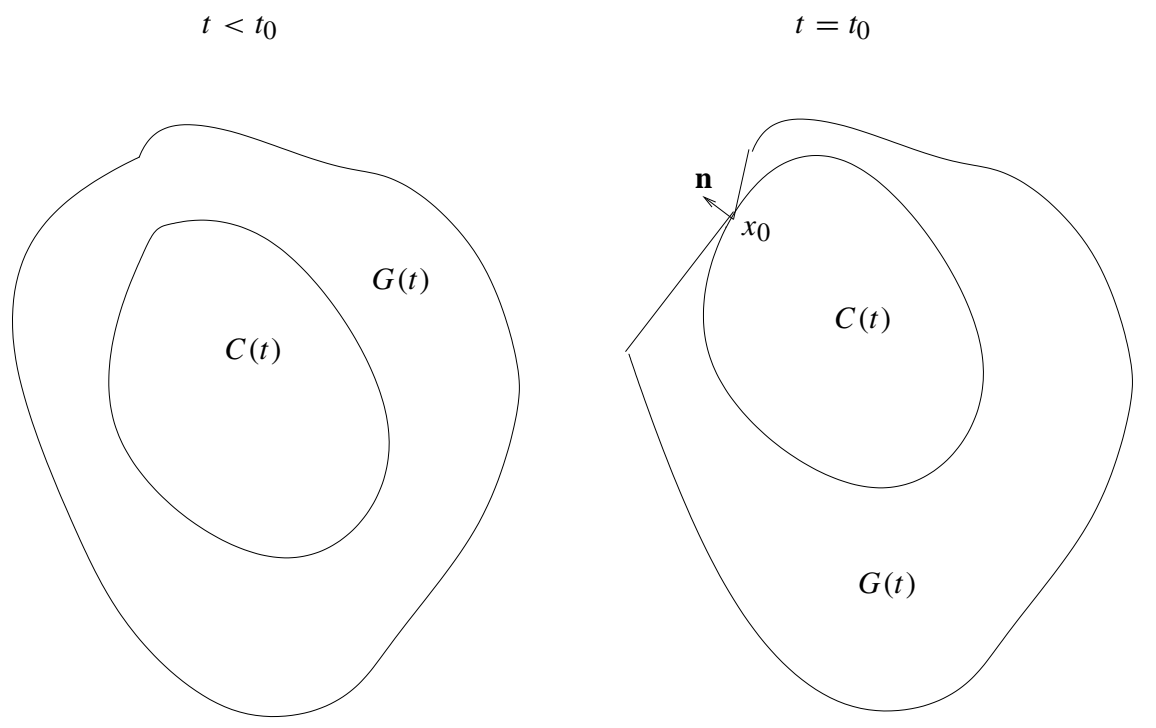

FIG. 1. The touching condition for a supresolution.

To see this, suppose that $C(t)$ satisfies the condition (1) for supersolutions, for example, and let $C_{\delta}(t)=\left\{x \in \mathbb{R}^{n}: d_{C(t)}(x)>\delta\left(\left|x-x_{0}\right|^{2}+\left|t-t_{0}\right|^{2}\right)\right\}$ for $\delta>0$. Since $C(t)$ is smooth, $C_{\delta}(t)$ is smooth at least near $\left(x_{0}, t_{0}\right)$ and $C_{\delta}(t) \subset C(t)$ for small $\delta>0$. Since we are only interested in local behavior near $\left(x_{0}, t_{0}\right)$, we may assume $C_{\delta}$ is smooth globally. Moreover,

$$
\partial C_{\delta}(t) \cap \partial G(t)= \begin{cases}\left\{x_{0}\right\} & \text { if } t=t_{0} \\ \emptyset & \text { if } t \neq t_{0}\end{cases}
$$

By Definition 3.1 the velocity of $\partial C_{\delta}(t)$ at $\left(x_{0}, t_{0}\right)$ is $\geqslant v(\mathbf{n})$ for every $\delta>0$. Now let $\delta \rightarrow 0$ to get (3.1). By analogy to viscosity solutions in PDEs, the condition 3.1) is similar to the use of extrema instead of strict extrema.

In [5] Bellettini and Novaga studied a wide class of geometric evolution problems in terms of minimal barriers. The following comes from Definition 2.1 in [5]. We denote by $\mathcal{P}\left(\mathbb{R}^{n}\right)$ the set of all subsets of $\mathbb{R}^{n}$.

DEFINITION 3.3 Let $\mathcal{F}$ be a family of functions with the following property: for any $f \in \mathcal{F}$ there exist $a, b \in \mathbb{R}, a<b$, such that $f:[a, b] \rightarrow \mathcal{P}\left(\mathbb{R}^{n}\right)$. A function $\phi$ is a barrier with respect to $\mathcal{F}$ if and only if there exists a convex set $L \subseteq I:=[0, \infty)$ such that $\phi: L \rightarrow \mathcal{P}\left(\mathbb{R}^{n}\right)$ and if $f:[a, b] \subseteq L \rightarrow \mathcal{P}\left(\mathbb{R}^{n}\right)$ belongs to $\mathcal{F}$ and $f(a) \subseteq \phi(a)$ then $f(b) \subseteq \phi(b)$.

REMARK 3.4 We show that a subsolution or a supersolution in the sense of Definition 3.1 is a barrier with respect to an appropriate family $\mathcal{F}$. We present the argument only for subsolutions since the case of supersolutions is similar. Let $\{G(t)\}_{t} \geqslant 0$ be a subsolution in the sense of Definition 3.1 . Let $\mathcal{F}$ be the collection of all smoothly evolving open sets $\{C(t)\}_{t} \geqslant 0$ such that

$$
k_{\partial C(t)}<v(\mathbf{n}),
$$


where $\mathbf{n}$ is normal to $\partial C(t)$. We want to show that $\{G(t)\}_{t \geqslant 0}$ is a barrier with respect to $\mathcal{F}$. In fact, if not, there exist $t_{0}<t_{1}$ and $\{C(t)\}_{t} \geqslant 0 \in \mathcal{F}$ such that

$$
C\left(t_{0}\right) \subset G\left(t_{0}\right), \text { but } C\left(t_{1}\right) \nsubseteq G\left(t_{1}\right) .
$$

In view of [3.4), there exist $t^{*}$ and $\epsilon>0$ such that $C(t) \subset G(t)$ for all $t \in\left(t^{*}-\epsilon, t^{*}\right]$ and $\partial C\left(t^{*}\right) \cap \partial G\left(t^{*}\right) \neq \emptyset$. Fix $x_{0} \in \partial C\left(t^{*}\right) \cap \partial G\left(t^{*}\right)$. Since $G(t)$ is a subsolution in the sense of Definition 3.1 we can conclude, in view of Remark 3.2 that the velocity of $\partial C\left(t^{*}\right)$ at $x_{0}$ is at least $v(\mathbf{n})$, which contradicts $C(t) \in \mathcal{F}$.

The following lemma shows that Definition 3.1 gives a reasonable notion of weak solutions for geometric evolutions.

Lemma 3.5 If $G(t)$ is a supersolution of the geometric evolution $V=v(\mathbf{n})$ and if $\partial G(t)$ is $C^{1}$ at $\left(x_{0}, t_{0}\right)$, then

$$
k_{\partial G(t)}\left(x_{0}, t_{0}\right) \geqslant v(\mathbf{n}) .
$$

A similar result holds for a subsolution with reverse inequality.

Proof. Since $\partial G(t)$ is $C^{1}$ at $\left(x_{0}, t_{0}\right)$, after a rotation, there exist $r>0$ and a $C^{1}$ function $g$ such that

$$
G \cap B_{r}\left(x_{0}, t_{0}\right)=\left\{(x, t) \in B_{r}\left(x_{0}, t_{0}\right): x_{n}>g\left(x^{\prime}, t\right), x^{\prime} \in \mathbb{R}^{n-1}\right\} .
$$

Then for small $\delta>0$, there exists $G_{\delta}(t)$ (see Remark 3.2 for a simple construction) such that

$$
\begin{aligned}
& G_{\delta}(t) \rightarrow G(t) \cap B_{r}\left(x_{0}, t_{0}\right) \text { in Hausdorff metric, and } \\
& \text { (1), (2) in Definition 3.1 hold. }
\end{aligned}
$$

Since $\partial G(t)$ is $C^{1}$ at $\left(x_{0}, t_{0}\right)$ and this is a local property, we may assume that $G_{\delta}(t)$ is a $C^{1}$ smoothly evolving set. By Definition 3.1 the velocity of $\partial G_{\delta}(t)$ at $\left(x_{0}, t_{0}\right)$ is greater than $v(\mathbf{n})$ for every $\delta>0$. Let $\delta \rightarrow 0$ to get the result.

\section{The proof of Theorem 1.1}

In this section, we give the proof of Theorem 1.1. The crucial step in the proof is the following lemma.

LEMMA 4.1 Let $\mathcal{A}(t)$ satisfy

(A1) Semigroup: $\mathcal{A}(t+s)=\mathcal{A}(t) \mathcal{A}(s)$ for all $s, t \geqslant 0$;

(A2) Translation invariance: $\mathcal{A}(t) \tau_{y}=\tau_{y} \mathcal{A}(t)$ for all $t \geqslant 0$ and $y \in \mathbb{R}^{n}$;

(A3) Monotonicity: If $G \subset H$, then $\mathcal{A}(t) G \subset \mathcal{A}(t) H$;

(A4) Finite propagation speed: Let $\mathcal{A}(t) \emptyset=\emptyset, \mathcal{A}(t) \mathbb{R}^{n}=\mathbb{R}^{n}$ for all $t>0$. There exists $c>0$ such that if $G \cap B_{c t}(x)=H \cap B_{c t}(x)$, then

$$
(\mathcal{A}(s) G) \cap B_{c(t-s)}(x)=(\mathcal{A}(s) H) \cap B_{c(t-s)}(x), \quad \forall s \leqslant t .
$$

Then $\mathcal{A}(t)$ maps half-planes to half-planes. Moreover, there exists a continuous function $v: S^{n-1} \rightarrow$ $\mathbb{R}$ such that $\{\mathcal{A}(t) G\}_{t \geqslant 0}$ is a weak solution of $V=v(\mathbf{n})$ whenever $G$ is a half-plane. 
Proof. 1. Fix some $t>0$ and let $\mathbf{n} \in S^{n-1}$. By finite propagation speed (A4), there exists $y_{\mathbf{n}} \in \mathbb{R}^{n}$ such that

$$
\mathcal{A}(t) H_{\mathbf{n}} \subset \tau_{y_{\mathbf{n}}} H_{\mathbf{n}}
$$

Define

$$
d(\mathbf{n}, t)=\inf \left\{y_{\mathbf{n}} \cdot \mathbf{n}: \mathcal{A}(t) H_{\mathbf{n}} \subset \tau_{y_{\mathbf{n}}} H_{\mathbf{n}}\right\} .
$$

If we choose $y=d(\mathbf{n}, t) \mathbf{n}$, then (4.1) implies $\mathcal{A}(t) H_{\mathbf{n}} \subset \tau_{y} H_{\mathbf{n}}$. For any $x$ such that $x \cdot \mathbf{n} \leqslant 0$, we have $\tau_{x} H_{\mathbf{n}} \subset H_{\mathbf{n}}$ since $H_{\mathbf{n}}$ is open. Hence, by translation invariance (A2) and monotonicity (A3),

$$
\tau_{x} \mathcal{A}(t) H_{\mathbf{n}} \subset \mathcal{A}(t) H_{\mathbf{n}}
$$

This implies $\mathcal{A}(t) H_{\mathbf{n}}$ is a half-plane with normal $\mathbf{n}$. But by the definition of $y$, we have $\mathcal{A}(t) H_{\mathbf{n}}=$ $\tau_{y} H_{\mathbf{n}}$.

2. We define $v(\mathbf{n})$ by the relation

$$
\mathcal{A}(t) H_{\mathbf{n}}=\tau_{t v(\mathbf{n}) \mathbf{n}} H_{\mathbf{n}} .
$$

First, we claim that there exists $C>0$ such that

$$
|v(\mathbf{n})| \leqslant C, \quad \forall \mathbf{n} \in S^{n-1}
$$

Suppose that 4.3) does not hold. Then for given $c>0$ in (A4), there exists $\mathbf{n} \in S^{n-1}$ such that $|v(\mathbf{n})|>2 c$. We may assume $v(\mathbf{n})>0$. For $H_{\mathbf{n}}=\left\{y \in \mathbb{R}^{n}: y \cdot \mathbf{n}<a\right\}$, fix $x \in \mathbb{R}^{n}$ such that $x \cdot \mathbf{n}=v(\mathbf{n})+a$. Then $H_{\mathbf{n}} \cap B_{2 c}(x)=\emptyset$.

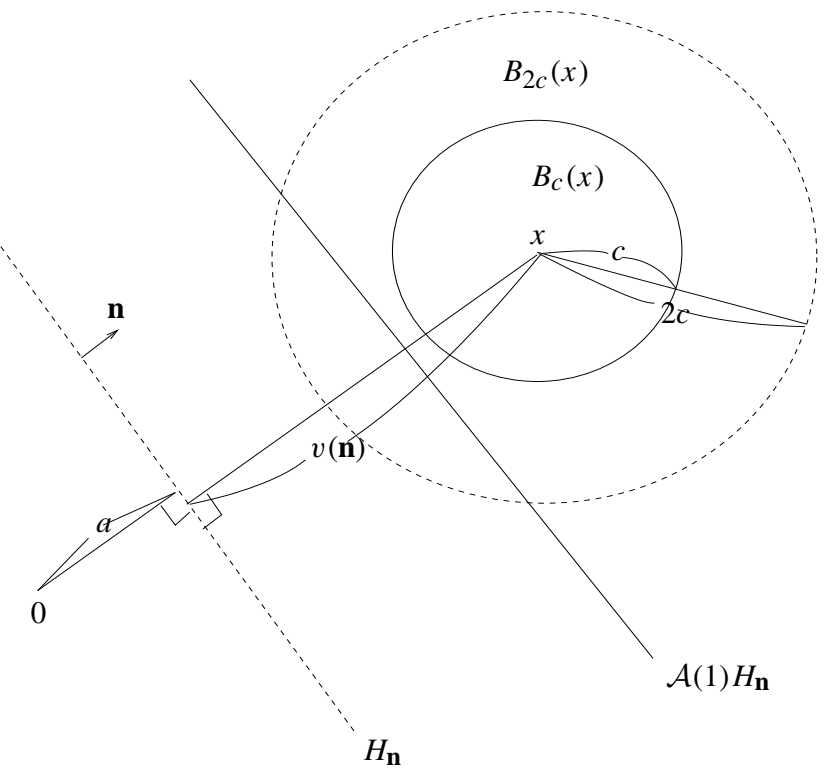

FIG. 2. The bound of $v(\mathbf{n})$. 
By finite propagation speed (A4), $\left(\mathcal{A}(1) H_{\mathbf{n}}\right) \cap B_{c}(x)=(\mathcal{A}(1) \emptyset) \cap B_{c}(x)=\emptyset$. This implies $x \notin \mathcal{A}(1) H_{\mathbf{n}}$ and hence $v(\mathbf{n})=x \cdot \mathbf{n}-a \leqslant 2 c$, which is a contradiction, and hence $v$ is bounded. Note that so far we have made no use of (A1).

For $y=v(\mathbf{n}) \mathbf{n}$, we have $\mathcal{A}(1) H_{\mathbf{n}}=\tau_{y} H_{\mathbf{n}}$. We claim $\mathcal{A}(t) H_{\mathbf{n}}=\tau_{t y} H_{\mathbf{n}}$. By semigroup property (A1), we have, for any $h>0$,

$$
\mathcal{A}(n h) H_{\mathbf{n}}=\mathcal{A}(h)^{n} H_{\mathbf{n}} .
$$

In particular, $\mathcal{A}(1 / n) H_{\mathbf{n}}=\tau_{(1 / n) y} H_{\mathbf{n}}, n \in \mathbb{N}$. By finite propagation speed (A4), we have

$$
\rho\left(\mathcal{A}(h) H_{\mathbf{n}}, H_{\mathbf{n}}\right) \leqslant C h .
$$

Fix any $n \in \mathbb{N}$. Then we choose $m \in \mathbb{N}$ such that $m / n \leqslant t<(m+1) / n$. Without loss of generality, we may assume

$$
\mathcal{A}\left(\frac{m}{n}\right) H_{\mathbf{n}} \subset \mathcal{A}(t) H_{\mathbf{n}} \subset \mathcal{A}\left(\frac{m+1}{n}\right) H_{\mathbf{n}} .
$$

Then 4.6 implies

$$
\begin{aligned}
\rho\left(\mathcal{A}(t) H_{\mathbf{n}}, \tau_{t y} H_{\mathbf{n}}\right) & \leqslant \rho\left(\mathcal{A}(t) H_{\mathbf{n}}, \mathcal{A}\left(\frac{1}{n}\right)^{m} H_{\mathbf{n}}\right)+\rho\left(\mathcal{A}\left(\frac{1}{n}\right)^{m} H_{\mathbf{n}}, \tau_{t y} H_{\mathbf{n}}\right) \\
& \leqslant 2 \rho\left(\mathcal{A}\left(\frac{1}{n}\right)^{m} H_{\mathbf{n}}, \mathcal{A}\left(\frac{1}{n}\right)^{m+1} H_{\mathbf{n}}\right) \leqslant \frac{2}{n}|v(\mathbf{n})| \rightarrow 0 \quad \text { as } n \rightarrow \infty
\end{aligned}
$$

Hence $H_{\mathbf{n}}$ has constant speed $v(\mathbf{n})$ along normal $\mathbf{n}$.

3. Now we show that the function $v$ is continuous. For given open subsets $G, H$, by finite propagation speed (A4), there exists $R>0$ such that if $G \cap B_{2 R}(0)=H \cap B_{2 R}(0)$, then $(\mathcal{A}(1) G) \cap B_{R}(0)=(\mathcal{A}(1) H) \cap B_{R}(0)$. By $\left.\sqrt{4.3}\right)$, we may assume $R \geqslant 4 C$. Fix $\mathbf{n} \in S^{n-1}$. We need to estimate $\left|v(\mathbf{n})-v\left(\mathbf{n}^{\prime}\right)\right|$ for $\mathbf{n}^{\prime} \in S^{n-1}$ close to $\mathbf{n}$ (see Figure 3). In particular, we can restrict our attention to $\mathbf{n}^{\prime}$ such that

$$
1-\epsilon \leqslant\left|\mathbf{n} \cdot \mathbf{n}^{\prime}\right|<1,
$$

with $\epsilon$ to be chosen later. Given such an $\mathbf{n}^{\prime}$, we define

$$
H_{\mathbf{n}^{\prime}}^{-}=\left\{x \in B_{2 R}(0): x \cdot \mathbf{n}^{\prime}<a^{-}\right\},
$$

where we select $a^{-}$to be the smallest number such that

$$
x \in B_{2 R}(0), x \cdot \mathbf{n}^{\prime}>a^{-} \Rightarrow x \cdot \mathbf{n}>0 .
$$

A simple calculation shows that

$$
a^{-}=2 R \sqrt{1-\left(\mathbf{n} \cdot \mathbf{n}^{\prime}\right)^{2}}
$$

In particular, the choice of $a^{-}$implies that

$$
H_{\mathbf{n}} \cap B_{2 R}(0) \subset H_{\mathbf{n}^{\prime}}^{-} \cap B_{2 R}(0) .
$$

As a result, we know that

$$
\mathcal{A}(1) H_{\mathbf{n}} \subset \mathcal{A}(1) H_{\mathbf{n}^{\prime}}^{-} \quad \text { in } B_{R}(0) .
$$




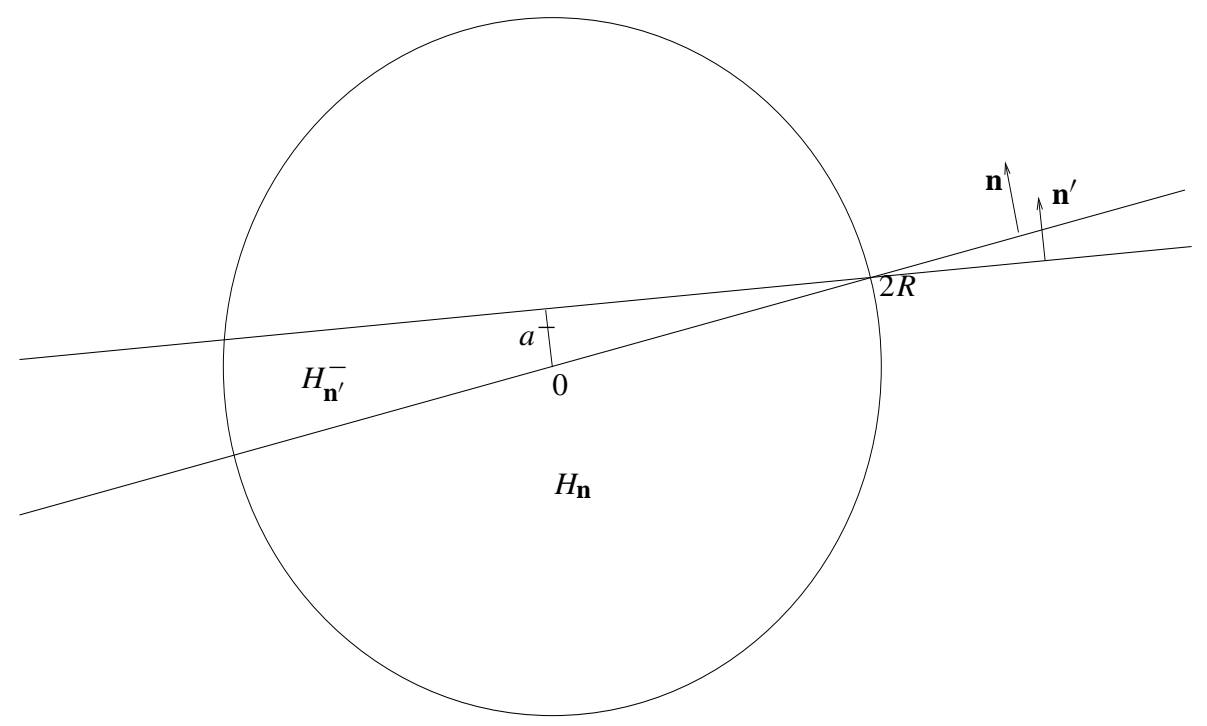

FIG. 3. $H_{\mathbf{n}}$ and $H_{\mathbf{n}^{\prime}}^{-}$

Let

$$
\sigma_{0}=\inf \left\{\sigma \in \mathbb{R}: \sigma \mathbf{n} \notin \mathcal{A}(1) H_{\mathbf{n}^{\prime}}^{-}\right\} .
$$

We try to find $\sigma_{0}$. In fact, $\mathcal{A}(1) H_{\mathbf{n}^{\prime}}^{-}=\left\{x \in \mathbb{R}^{n}: x \cdot \mathbf{n}^{\prime}<a^{-}+v\left(\mathbf{n}^{\prime}\right)\right\}$. Hence for any $\sigma \geqslant \sigma_{0}$, we have $\sigma \mathbf{n} \cdot \mathbf{n}^{\prime} \geqslant a^{-}+v\left(\mathbf{n}^{\prime}\right)$. Therefore

$$
\sigma_{0}=\frac{1}{\mathbf{n} \cdot \mathbf{n}^{\prime}}\left(a^{-}+v\left(\mathbf{n}^{\prime}\right)\right) .
$$

In view of (4.7) and (4.9), we have

$$
\left|\sigma_{0} \mathbf{n}\right| \leqslant \frac{1}{\left|\mathbf{n} \cdot \mathbf{n}^{\prime}\right|}\left(2 R \sqrt{1-\left(\mathbf{n} \cdot \mathbf{n}^{\prime}\right)^{2}}+C\right) \leqslant R\left(\frac{2 \sqrt{2 \epsilon-\epsilon^{2}}+1 / 4}{1-\epsilon}\right) .
$$

Since the right-hand side in (4.11) is less than $R$ for small $\epsilon>0$ we may assume $\sigma_{0} \mathbf{n} \in B_{R}(0)$. Thus we find by 4.10 ,

$$
v(\mathbf{n})=\inf \left\{\sigma \in \mathbb{R}: \sigma \mathbf{n} \notin \mathcal{A}(1) H_{\mathbf{n}}\right\} \leqslant \inf \left\{\sigma \in \mathbb{R}: \sigma \mathbf{n} \notin \mathcal{A}(1) H_{\mathbf{n}^{\prime}}^{-}\right\} \leqslant \frac{1}{\mathbf{n} \cdot \mathbf{n}^{\prime}}\left(a^{-}+v\left(\mathbf{n}^{\prime}\right)\right) .
$$

Next choose $H_{\mathbf{n}^{\prime}}^{+}=\left\{x \in B_{2 R}(0): x \cdot \mathbf{n}^{\prime}<a^{+}\right\}$in place of 4.8 where $a^{+}$is the greatest number such that

$$
x \in B_{2 R}(0), x \cdot \mathbf{n}^{\prime}<a^{+} \Rightarrow x \cdot \mathbf{n}<0 .
$$

Applying the previous argument, we have

$$
v(\mathbf{n}) \geqslant \frac{1}{\mathbf{n} \cdot \mathbf{n}^{\prime}}\left(-a^{+}+v\left(\mathbf{n}^{\prime}\right)\right) .
$$


Combining these two inequalities, we conclude

$$
\frac{1}{\mathbf{n} \cdot \mathbf{n}^{\prime}}\left(-a^{+}+v\left(\mathbf{n}^{\prime}\right)\right) \leqslant v(\mathbf{n}) \leqslant \frac{1}{\mathbf{n} \cdot \mathbf{n}^{\prime}}\left(a^{-}+v\left(\mathbf{n}^{\prime}\right)\right) .
$$

The continuity of $v(\mathbf{n})$ follows from (4.9) and (4.12).

4. Finally, if $G$ is a half-plane, then $\{\mathcal{A}(t) G\}_{t} \geqslant 0$ is both a subsolution and a supersolution of $V=v(\mathbf{n})$ since $\mathcal{A}(t) G$ itself is smooth for all $t \geqslant 0$ and the normal velocity is $v(\mathbf{n})$, where $\mathbf{n}$ is the normal to $\partial G$.

We now turn to the proof of our first main result.

Proof of Theorem 1.1 1. We only prove the supersolution case since the other case can be proved similarly. So write $\mathcal{A}(t)=\mathcal{A}_{1}(t)$. Suppose $\mathcal{A}(t)$ satisfies (A2)-(A4) and

$$
\mathcal{A}(t) \mathcal{A}(s) \subset \mathcal{A}(t+s) \quad \forall t, s \geqslant 0 .
$$

The existence of a continuous function $v$ follows from Lemma 4.1 (careful inspection of the proof of Lemma 4.1 tells us that we do not need the property (A1) for the existence of $v$ ).

2. To prove $\{\mathcal{A}(t) G\}_{t} \geqslant 0$ is a supersolution of $V=v(\mathbf{n})$, fix any smoothly evolving set $\{C(t)\}_{t} \geqslant 0$ such that for an open subset $G$ in $\mathbb{R}^{n}, C(t) \subset \mathcal{A}(t) G$ for all $t \in\left(t_{0}-\epsilon, t_{0}\right]$ for small $\epsilon>0$ and

$$
\partial C(t) \cap \partial(\mathcal{A}(t) G)= \begin{cases}\left\{x_{0}\right\} & \text { if } t=t_{0}, \\ \varnothing & \text { if not. }\end{cases}
$$

Let $w$ denote the normal velocity of $C\left(t_{0}\right)$ at $x_{0}$, and suppose toward a contradiction that $w<v(\mathbf{n})$. Since $C(t)$ is a smoothly evolving set, after a change of coordinates, we may as well assume that near $\left(x_{0}, t_{0}\right), \partial C(t)$ is the graph of a smooth function $g\left(x^{\prime}, t\right), x^{\prime} \in \mathbb{R}^{n-1}$, such that

$$
\mathbf{n}=e_{n}, \quad x_{0}=0, \quad g\left(0, t_{0}\right)=0, \quad D g\left(0, t_{0}\right)=0 .
$$

Then we can choose small $h>0$ and $c>0$ such that, by expansion, we have

$$
\left|g\left(x^{\prime}, t_{0}-h\right)+w h\right| \leqslant k h^{2} \quad \text { for some } k \text {, and all } x^{\prime} \in B_{2 c h}(0) .
$$

Hence $\tau_{y_{1}} H_{\mathbf{n}} \subset\left\{g\left(x^{\prime}, t_{0}-h\right)>x_{n}\right\} \subset \mathcal{A}\left(t_{0}-h\right) G$ in $B_{2 c h}(0)$, where $y_{1} \cdot \mathbf{n}=-w h-k h^{2}$. Utilizing monotonicity (A3) and finite propagation speed (A4), we find

$$
\mathcal{A}(h) \tau_{y_{1}} H_{\mathbf{n}} \subset \mathcal{A}(h) \mathcal{A}\left(t_{0}-h\right) G \subset \mathcal{A}\left(t_{0}\right) G \quad \text { in } B_{c h}(0),
$$

where we used 44.13 in the second inclusion. Notice that $\mathcal{A}(h) \tau_{y_{1}} H_{\mathbf{n}}=\left\{x \cdot \mathbf{n}<(v(\mathbf{n})-w) h-k h^{2}\right\}$. Hence $(v(\mathbf{n})-w) h<k h^{2}$ according to 4.14). Since $h>0$ can be arbitrarily small, we have a contradiction.

3. If $\mathcal{A}(t)$ satisfies (A1)-(A4), then steps 1 and 2 imply that there exist $v_{1}, v_{2}: S^{n-1} \rightarrow \mathbb{R}$ such that for every open $G,\{\mathcal{A}(t) G\}_{t} \geqslant 0$ is a supersolution of $V=v_{1}(\mathbf{n})$ and a subsolution of $V=v_{2}(\mathbf{n})$. If $v_{1}(\mathbf{n}) \neq v_{2}(\mathbf{n})$ for some $\mathbf{n} \in S^{n-1}$, then

$$
\mathcal{A}(1) H_{n}=\left\{x \in \mathbb{R}^{n}: x \cdot \mathbf{n}<v_{1}(\mathbf{n})\right\}=\left\{x \in \mathbb{R}^{n}: x \cdot \mathbf{n}<v_{2}(\mathbf{n})\right\},
$$

which is impossible.

The following result is a direct consequence of Lemma 3.5 and Theorem 1.1 which shows that the velocity motion is classical as long as the evolution is smooth. 
COROLlaRY 4.2 Suppose that $\mathcal{A}(t)$ satisfies the hypotheses (A1)-(A4). Then there exists a continuous function $v: S^{n-1} \rightarrow \mathbb{R}$ such that for every open set $G,\{\mathcal{A}(t) G\}_{t} \geqslant 0$ is a classical solution of $V=v(\mathbf{n})$ whenever its boundary is $C^{1}$.

\section{Threshold dynamics on $\mathbb{R}^{n}$}

In this section, we consider threshold type dynamics on $\mathbb{R}^{n}$ introduced by Gravner and Griffeath [11]. In Section 2 of [13], Ishii et al. studied approximation schemes for curvature-independent motion by using threshold type dynamics. We consider a more general class of threshold type dynamics. We do not make any assumptions about the detailed structure of dynamics. Rather, we merely assume simple properties: monotonicity, translation invariance and finite propagation speed. We recall the hypotheses (F1)-(F4) from the Introduction. For $h>0$, suppose that $F_{h}$ is a mapping from the set of open subsets in $\mathbb{R}^{n}$ to itself such that

(F1) Monotonicity: If $G \subset H \subset \mathbb{R}^{n}$, then $F_{h} G \subset F_{h} H$;

(F2) Translation invariance: $F_{h}\left(\tau_{x} G\right)=\tau_{x} F_{h} G$, for all $x \in \mathbb{R}^{n}$;

(F3) Finite propagation speed: There exists $c>0$ such that if $G \cap B_{2 c h}(x)=H \cap B_{2 c h}(x)$, then

$$
F_{h} G \cap B_{c h}(x)=F_{h} H \cap B_{c h}(x) ;
$$

(F4) For any $s \leqslant h$ and $\mathbf{n} \in S^{n-1}$, there exists $C>0$ such that

$$
\rho\left(F_{h} H_{\mathbf{n}}, F_{s}^{\lfloor h / s\rfloor} H_{\mathbf{n}}\right) \leqslant C|h-\lfloor h / s\rfloor s| .
$$

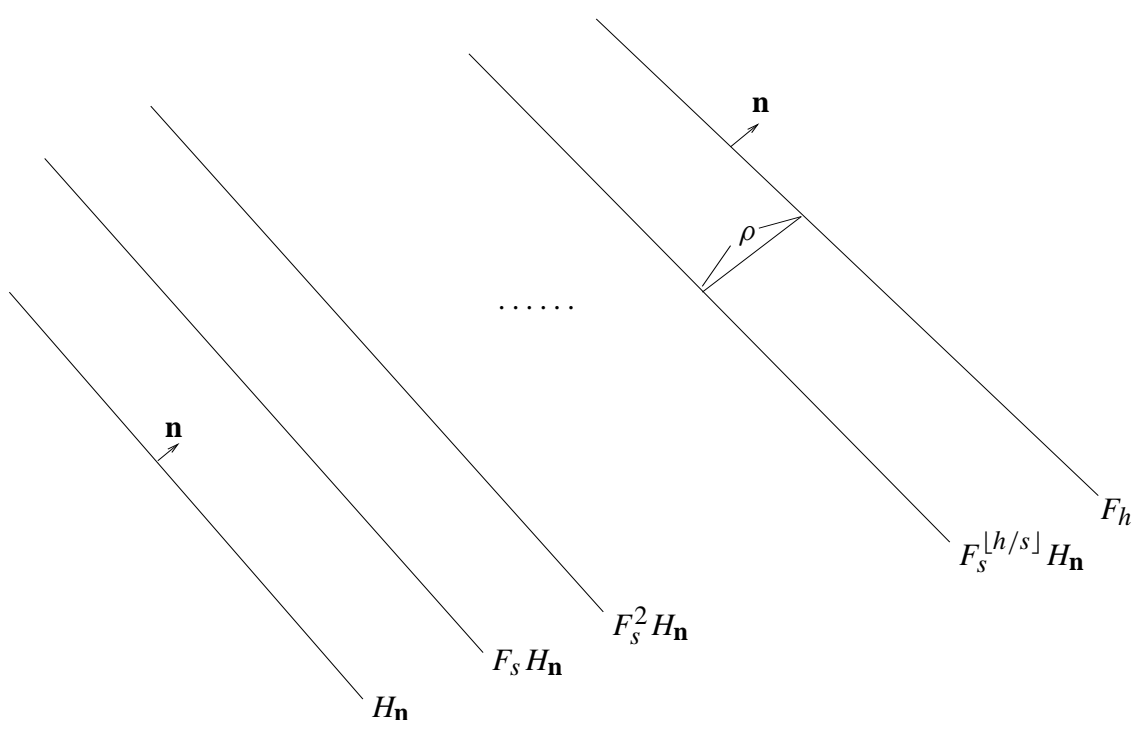

FIG. 4. The distance between $F_{h} H_{\mathbf{n}}$ and $F_{s}^{\lfloor h / s\rfloor} H_{\mathbf{n}}$.

The assumption (F4) may appear unusual. (F1)-(F3) are the conditions for single parameter $h$. It is reasonable to compare the evolution between two parameters. The assumption (F4) together with (F3) gives us some control on at least the evolution of half-planes. 
For given $t \geqslant 0$ and an open subset $G$ in $\mathbb{R}^{n}$, we define

$$
\begin{aligned}
& \underline{\mathcal{A}}(t) G=\left(\bigcup_{G^{\prime} \subset \subset G} \liminf _{h \rightarrow 0} F_{h}^{\lfloor t / h\rfloor} G^{\prime}\right)^{o}, \\
& \overline{\mathcal{A}}(t) G=\left(\bigcap_{G^{\prime} \supset \supset G} \limsup _{h \rightarrow 0} F_{h}^{\lfloor t / h\rfloor} G^{\prime}\right)^{o},
\end{aligned}
$$

where $A^{o}$ denotes the interior of the set $A$ and

$$
\begin{aligned}
& \liminf _{h \rightarrow 0} F_{h}^{\lfloor t / h\rfloor}=\bigcup_{h>0} \bigcap_{0<s \leqslant h} F_{s}^{\lfloor t / s\rfloor}, \\
& \limsup _{h \rightarrow 0} F_{h}^{\lfloor t / h\rfloor}=\bigcap_{h>0} \bigcup_{0<s \leqslant h} F_{s}^{\lfloor t / s\rfloor} .
\end{aligned}
$$

In 5.3, if $G$ is not bounded, one can modify the definition to make $\overline{\mathcal{A}}(t)$ well defined. For example, one can take the intersection of $G^{\delta} \searrow G$ where $G^{\delta}=\left\{x \in \mathbb{R}^{n}: \operatorname{dist}(x, G)<\delta\right\}$. First we prove the following

LEMMA 5.1 If $G_{1} \subset G_{2}$ then

$$
\varrho\left(F_{h}\left(G_{1}\right), F_{h}\left(G_{2}\right)\right) \geqslant \varrho\left(G_{1}, G_{2}\right)
$$

where $\varrho(G, H)=\min \left\{\operatorname{dist}\left(x, H^{c}\right), \operatorname{dist}\left(y, G^{c}\right): x \in G, y \in H\right\}$. Similarly,

$$
\varrho\left(\liminf _{h \rightarrow 0} F_{h}^{[t / h]} G_{1}, \liminf _{h \rightarrow 0} F_{h}^{[t / h]} G_{2}\right) \geqslant \varrho\left(G_{1}, G_{2}\right) .
$$

Proof. The lemma follows from noting that if $G$ and $H$ are open and $G \subset H$ then

$$
\varrho(G, H) \geqslant a \quad \text { if and only if } \quad \tau_{y} G \subset H \text { for all }|y| \leqslant a .
$$

In particular, for $G_{1} \subset G_{2}$, if we write $d:=\varrho\left(G_{1}, G_{2}\right)$ then the above implies that $\tau_{y} G_{1} \subset G_{2}$ for all $|y| \leqslant d$. Then monotonicity (F1) and translation invariance (F2) imply that $\tau_{y} F_{h} G_{1} \subset F_{h} G_{2}$ for all $|y| \leqslant d$, and again using (5.6), we deduce the first conclusion of the lemma. The second conclusion follows by essentially the same argument, after noting that liminf is monotone in the sense that

$$
U_{h} \subset V_{h} \forall h>0 \Rightarrow \liminf U_{h} \subset \liminf V_{h} .
$$

We are now ready to give the proof of Theorem 1.2

Proof of Theorem 1.2. 1. The hypotheses (A2)-(A3) directly follow from (F1)-(F2). To prove (A4), fix a constant $c>0$ in (F3). Suppose that $G \cap B_{c t}(x)=H \cap B_{c t}(x), G, H \subset \mathbb{R}^{n}, t>0$. Fix any $s \leqslant t$. By finite propagation speed (F3) and iterations, we have for any $h>0$,

$$
F_{h}^{\lfloor s / h\rfloor} G \cap B_{c(t-\lfloor s / h\rfloor h)}(x)=F_{h}^{\lfloor s / h\rfloor} H \cap B_{c(t-\lfloor s / h\rfloor h)}(x) .
$$

Since $\lfloor s / h\rfloor h \leqslant s$ for any $h>0$, we get

$$
F_{h}^{\lfloor s / h\rfloor} G \cap B_{c(t-s)}(x)=F_{h}^{\lfloor s / h\rfloor} H \cap B_{c(t-s)}(x) .
$$

Then (A4) follows by taking lim sup in 5.77 . 
2. We only prove $\underline{\mathcal{A}}(t) \underline{\mathcal{A}}(s) \subset \underline{\mathcal{A}}(s+t)$, since the other case is similar. Fix an open set $G \subset$ $\mathbb{R}^{n}$ and $s, t>0$. We may assume that $G$ is bounded, because if not, we can prove the result for $G \cap B_{R}(0)$ for arbitrary $R>0$, and then use finite propagation speed (F3) to deduce that

$$
\underline{\mathcal{A}}(t) \underline{\mathcal{A}}(s) G=\bigcup_{R>0} \underline{\mathcal{A}}(t) \underline{\mathcal{A}}(s)\left(G \cap B_{R}(0)\right) \subset \bigcup_{R>0} \underline{\mathcal{A}}(s+t)\left(G \cap B_{R}(0)\right)=\underline{\mathcal{A}}(s+t) G .
$$

Let $H=\underline{\mathcal{A}}(s) G$, and note that since $G$ is assumed to be bounded, finite propagation speed implies that $H$ is bounded. Our goal is now to show that $\underline{\mathcal{A}}(t) H \subset \underline{\mathcal{A}}(t+s) G$. Toward this end, we fix any $H^{\prime} \subset \subset H$, and we let

$$
G_{k}:=\{x \in G: \operatorname{dist}(x, \partial G)>1 / k\}, \quad H_{k}=\left(\bigcup_{h>0} \bigcap_{0<h^{\prime}<h} F_{h^{\prime}}^{\left[s / h^{\prime}\right]} G_{k}\right)^{o} .
$$

It then follows from the definitions that $G=\bigcup_{k} G_{k}$ and $H=\bigcup_{k} H_{k}$. It also follows from the above lemma that $\varrho\left(H_{j}, H_{k}\right) \geqslant \varrho\left(G_{j}, G_{k}\right)$. We first claim that

$$
H^{\prime} \subset H_{k} \quad \text { for some } k \text {. }
$$

To prove this, suppose toward a contradiction that it does not hold. Then for each $k$ there exists some $x_{k} \in H^{\prime} \backslash H_{k}$. Upon passing to a subsequence, we may assume (since $H$ is bounded) that

$$
x_{k} \rightarrow x \in \bar{H}^{\prime} \subset H \text {. }
$$

Since $x \in H$, clearly $x$ must belong to $H_{k}$ for some $k$. Then $H_{k} \subset H_{k+1}$ and $\varrho\left(H_{k}, H_{k+1}\right) \geqslant$ $\varrho\left(G_{k}, G_{k+1}\right)=c_{k}>0$, and these together imply that $H_{k+1}$ contains a small ball $B_{c_{k}}(x)$. It follows that $B_{c_{k}}(x) \subset H_{j}$ for all $j \geqslant k+1$. Thus an open neighborhood of $x$ is contained in $H_{k}$. This however is impossible, in view of (5.9), and so we have provedthe claim.

3. Now fix $k$ satisfying 5.8 and note that

$$
\begin{aligned}
\underline{\mathcal{A}}(t+s) G & \supset \liminf _{h \rightarrow 0} F_{h}^{[(t+s) / h]} G_{k}=\liminf F_{h}^{[(t+s) / h]-[s / h]} F^{[s / h]} G_{k} \\
& \supset \liminf F_{h}^{[(t+s) / h]-[s / h]} H^{\prime}=\liminf F_{h}^{[t / h]} H^{\prime} .
\end{aligned}
$$

Since this holds for all $H^{\prime} \subset \subset H$, the definition of $\underline{\mathcal{A}}$ implies that $\underline{\mathcal{A}}(t+s) G \supset \underline{\mathcal{A}}(t) H$, as was to be proved.

4. By Theorem 1.1 there exist $\underline{v}, \bar{v}$ corresponding to $\underline{\mathcal{A}}(t), \overline{\mathcal{A}}(t)$ respectively such that for every open $G,\{\underline{\mathcal{A}}(t) G\}$ is a supersolution of $V=\underline{v}(\mathbf{n})$ and $\{\overline{\mathcal{A}}(t) G\}$ is a subsolution of $V=\bar{v}(\mathbf{n})$. Since $\underline{\mathcal{A}}(1) H_{\mathbf{n}}=\left\{x \in \mathbb{R}^{n}: x \cdot \mathbf{n}<\underline{v}(\mathbf{n})\right\} \subset \overline{\mathcal{A}}(1) \bar{H}_{\mathbf{n}}=\left\{x \in \mathbb{R}^{n}: x \cdot \mathbf{n}<\bar{v}(\mathbf{n})\right\}$, we have $\underline{v} \leqslant \bar{v}$.

To finish the remaining part, we need the following lemma.

LEMMA 5.2 Suppose (F1)-(F4) hold. Then

$$
\underline{\mathcal{A}}(t) H_{\mathbf{n}}=\overline{\mathcal{A}}(t) H_{\mathbf{n}} \quad \text { for each } t \geqslant 0 \text { and } \mathbf{n} \in S^{n-1} .
$$

Proof. Fix $s \leqslant h \leqslant t$ and $\mathbf{n} \in S^{n-1}$. Write

$$
\lfloor t / h\rfloor=m \leqslant\lfloor t / s\rfloor=n, \quad\lfloor h / s\rfloor=k, \quad k, m, n \in \mathbb{N} \cup\{0\} .
$$


Then (F4) and $F_{s} F_{h} H_{\mathbf{n}}=F_{h} F_{s} H_{\mathbf{n}}$ imply

$$
\begin{aligned}
\rho\left(F_{h}^{m} H_{\mathbf{n}}, F_{s}^{n} H_{\mathbf{n}}\right) \leqslant & \rho\left(F_{h}^{m} H_{\mathbf{n}}, F_{s}^{k} F_{h}^{m-1} H_{\mathbf{n}}\right)+\cdots+\rho\left(F_{s}^{k(m-1)} F_{h} H_{\mathbf{n}}, F_{s}^{k m} H_{\mathbf{n}}\right) \\
& +\rho\left(F_{s}^{k m} H_{\mathbf{n}}, F_{s}^{n} H_{\mathbf{n}}\right) \\
\leqslant & C\lfloor t / h\rfloor|h-\lfloor h / s\rfloor s|+\rho\left(F_{s}^{k m} H_{\mathbf{n}}, F_{s}^{n} H_{\mathbf{n}}\right) .
\end{aligned}
$$

Note that $k m \leqslant n$. We can estimate $\rho\left(F_{s}^{k m} H_{\mathbf{n}}, F_{s}^{n} H_{\mathbf{n}}\right)$ via finite propagation speed (F3) as follows:

$$
\begin{aligned}
\rho\left(F_{s}^{n} H_{\mathbf{n}}, F_{s}^{k m} H_{\mathbf{n}}\right) & \leqslant \rho\left(F_{s}^{n} H_{\mathbf{n}}, F_{s}^{n-1} H_{\mathbf{n}}\right)+\cdots+\rho\left(F_{s}^{k m+1} H_{\mathbf{n}}, F_{s}^{k m} H_{\mathbf{n}}\right) \\
& \leqslant C s(n-k m) \leqslant C(t-s k m) .
\end{aligned}
$$

Consider $s k m=s\lfloor h / s\rfloor\lfloor t / h\rfloor$. We may assume $k m \geqslant 1$ since $k=0$ or $m=0$ implies $\rho\left(F_{h}^{m} H_{\mathbf{n}}, F_{s}^{n} H_{\mathbf{n}}\right) \leqslant C|h-\lfloor h / s\rfloor s|$. Therefore we have

$$
s k m=s\lfloor h / s\rfloor\lfloor t / h\rfloor \rightarrow t \quad \text { as } s \leqslant h \rightarrow 0 .
$$

Hence we have a Cauchy estimate

$$
\rho\left(F_{h}^{\lfloor t / h\rfloor} H_{\mathbf{n}}, F_{s}^{\lfloor t / s\rfloor} H_{\mathbf{n}}\right) \rightarrow 0 \quad \text { as } h, s \rightarrow 0 .
$$

The conclusion follows from 5.10.

Let us show $\underline{v}=\bar{v}$ under the hypothesis (F4). Suppose $\underline{v}(\mathbf{n}) \neq \bar{v}(\mathbf{n})$ for some $\mathbf{n} \in S^{n-1}$. By Lemma 5.2. $\left\{\underline{\mathcal{A}}(t) H_{\mathbf{n}}\right\}_{t \geqslant 0}=\left\{\overline{\mathcal{A}}(t) H_{\mathbf{n}}\right\}_{t \geqslant 0}$ is a weak solution of $V=\underline{v}(\mathbf{n})$ and $V=\bar{v}(\mathbf{n})$. Then we have

$$
\overline{\mathcal{A}}(t) H_{\mathbf{n}}=\left\{x \in \mathbb{R}^{n}: x \cdot \mathbf{n}<\underline{v}(\mathbf{n})\right\}=\left\{x \in \mathbb{R}^{n}: x \cdot \mathbf{n}<\bar{v}(\mathbf{n})\right\},
$$

which is a contradiction.

REMARK 5.3 We notice that if (F4) does not hold, then we cannot guarantee the existence of a limiting velocity $v$. To see this, let $\left\{\mathcal{A}_{1}(t) G\right\}_{t \geqslant 0}$ and $\left\{\mathcal{A}_{2}(t) G\right\}_{t} \geqslant 0$ be families satisfying (A1)-(A4), and such that the associated velocity functions $v_{1}$ and $v_{2}$ (guaranteed by Theorem 1.1) satisfy

$$
v_{1}(\mathbf{n})<v_{2}(\mathbf{n}) \quad \forall \mathbf{n} \in S^{n-1} .
$$

We define

$$
F_{h} G= \begin{cases}\mathcal{A}_{1}(h) G & \text { if } 2^{-(j+1)} \leqslant h<2^{-j} \text { for } j \text { even } \\ \mathcal{A}_{2}(h) G & \text { if } 2^{-(j+1)} \leqslant h<2^{-j} \text { for } j \text { odd }\end{cases}
$$

Set

$$
\underline{\mathcal{A}}(t) G=\left(\bigcup_{G^{\prime} \subset \subset G} \liminf _{h \rightarrow 0} F_{h}^{\lfloor t / h\rfloor} G^{\prime}\right)^{o}, \quad \overline{\mathcal{A}}(t) G=\left(\bigcap_{G^{\prime} \supset \supset G} \limsup _{h \rightarrow 0} F_{h}^{\lfloor t / h\rfloor} G^{\prime}\right)^{o} .
$$

Since $v_{1}(\mathbf{n})<v_{2}(\mathbf{n})$, it is formally clear that

$$
\underline{\mathcal{A}}(t) G=\mathcal{A}_{1}(t) G, \quad \overline{\mathcal{A}}(t) G=\mathcal{A}_{2}(t) G \quad \forall t \geqslant 0 .
$$




\section{REFERENCES}

1. Alvarez, L., Guichard, F., Lions, P. L., \& Morel, J. M. Axioms and fundamental equations of image processing. Arch. Rat. Mech. Anal. 123 (1993), 199-257. Zbl 0788.68153 MR 1225209

2. BARles, G. \& Georgelin, C. A simple proof of convergence for an approximation scheme for computing motion by mean curvature. SIAM J. Numer. Anal. 32 (1995), 484-500. Zbl 0831.65138 MR 1324298

3. Barles, G. \& Souganidis, P. E. Convergence of approximation schemes for fully nonlinear second order equations. Asymptotic Anal. 4 (1991), 271-283. Zbl 0729.65077 MR 1115933

4. Barles, G. \& Souganidis, P. E. A new approach to front propagation problems: theory and application. Arch. Rat. Mech. Anal. 141 (1998), 237-296. Zbl 0904.35034 MR 1617291

5. Bellettini, G. \& Novaga, M. Comparison results between minimal barriers and viscosity solutions for geometric evolutions. Ann. Scuola Norm. Sup. Pisa Cl. Sci. (4) 26 (1998), 97-131. Zbl 0904.35041 MR 1632984

6. Bence, J., Merriman, B., \& Osher, S. Diffusion generated motion by mean curvature. Preprint (1992).

7. Biton, S. Nonlinear monotone semigroups and viscosity solutions. Ann. Inst. H. Poincaré Anal. Non Linéaire 18 (2001), 383-402. Zbl 1002.35060 MR 1831661

8. Chen, Y. G., Giga, Y., \& Goto, S. Uniqueness and existence of viscosity solutions of generalized mean curvature flow equations. J. Differential Geometry 33 (1991), 749-786. Zbl 0696.35087 MR 1100211

9. Evans, L. C. Convergence of an algorithm for mean curvature motion. Indiana Univ. Math. J. 42 (1993), 533-557. Zbl 0802.65098 MR 1237058

10. Evans, L. C. \& SPRUCK, J. Motion of level sets by mean curvature I. J. Differential Geometry 33 (1991), 635-681. Zbl 0726.53029 MR 1100206

11. Gravner, J. \& Griffeath, D. Threshold growth dynamics. Trans. Amer. Math. Soc. 340 (1993), $837-$ 870. Zbl 0791.58053 MR 1147400

12. GRAYSON, M. A short note on the evolution of surfaces via mean curvature. Duke Math. J. 58 (1989), 555-558. Zbl 0677.53059 MR 1016434

13. Ishit, H., Pires, G. E., \& Souganidis, P. E. Threshold dynamics type approximation schemes for propagation fronts. J. Math. Soc. Japan 51 (1999), 267-308. Zbl 0935.53006 MR 1674750

14. Sethian, J. A. Numerical algorithms for propagating interfaces: Hamilton-Jacobi equations and conservation laws. J. Differential Geometry 31 (1990), 131-161. 